\title{
A proposed reaction channel for the synthesis of the superheavy nucleus $\mathrm{Z}=109$.
}

\author{
K. Wang, ${ }^{1}$ Y. G. Ma,,${ }^{1}$, , 由 G. L. Ma,${ }^{1}$ Y. B. Wei, ${ }^{1}$ X. Z. Cai, ${ }^{1}$ J. G. Chen,${ }^{1}$ W. Guo, ${ }^{1}$ C. Zhong, ${ }^{1}$ and W. Q. Shen ${ }^{1,2,3}$ \\ ${ }^{1}$ Shanghai Institute of Applied Physics, Chinese Academy of Sciences, P. O. Box 800-204, Shanghai 201800 \\ ${ }_{2}$ CCAST (World Laboratory), P. O. Box 8730, Beijing 100080 \\ 3 Department of Physics, Ningbo University, Ningbo 315211
}

(Dated: Received 14 Nov 2003; Pubished in Chin. Phys. Lett. 21, 464 (2004))

\begin{abstract}
We apply a statistical-evaporation model (HIVAP) to calculate the cross sections of superheavy elements, mainly about actinide targets and compare with some available experimental data. A reaction channel ${ }^{30} \mathrm{Si}+{ }^{243} \mathrm{Am}$ is proposed for the synthesis of the element $\mathrm{Z}=109$ and the cross section is estimated.
\end{abstract}

PACS numbers: 25.70.Jj, 24.10.-i, 27.90.+b

The synthesis of superheavy elements always attract the attentions of the nuclear physicists and chemists since the superheavy island was predicted in 1960. Now it has been taken as one of the major research directions in main laboratories of nuclear physics around the world. So far, the heaviest elements $\mathrm{Z}=114$ and 116 were synthesized and identified in Dubna recently 1, 2]. Some structure and dynamical properties of heavy or superheavy nuclei have been investigated in various aspects 3, 4, 5, 6, 7, 8, 9]. However, theoretical efforts on the production cross section of superheavy elements are being waited for further improvement since most theoretical models are not able to predict the credible cross sections yet to provide the valid suggestions for the planning of experiments, hence the experiments have been performed more or less in the empirical ways.

Nevertheless, it is still interesting to do some semiempirical calculations to explore a possible way to help the experimental design. To this end, firstly, we perform the systematical calculations and let them agree with the experimental data well. Based on the good reproduction to some known data, we can make a reasonable extrapolation for the production cross section of some superheavy nuclei. In this paper we would like to explore this possibility with help of a statistical-evaporation model, socalled HIVAP, so that we can make a believable suggestion for the experimental proposal in our national laboratory of Heavy Ion Research Facility in Lanzhou (HIRFL).

HIVAP is a statistical-evaporation model 10, 11, 12, 13, 14], which assumed that the process of synthesizing superheavy nuclei includes two stages: firstly the projectile and target nuclei completely fuse to a compound nucleus, then the compound nucleus de-excites by fission or emitting light particles and $\gamma$-rays. The complete cross section $\sigma\left(E_{c m}\right)$ is assumed as

$$
\sigma\left(E_{c m}\right)=\pi \lambda^{2} \sum_{J=0}^{J_{\max }}(2 J+1) T\left(J, E_{c m}\right) P\left(J, E^{*}\right)
$$

where $\lambda$ is the de Broglie wave length of relative motion of the colliding nuclei, $E_{c m}$ is the energy of center-ofmass, $E^{*}$ is the excitation energy. $J$ is the total angular momentum quantum number, the upper limit $J_{\max }$ is obtained by 22. $T\left(J, E_{c m}\right)$ is the fusion probabilities of the $J$ th partial wave through the Coulomb barrier. $P\left(J, E_{c m}\right)$ is the survival probability of the residue evaporation nucleus after the compound nucleus de-excites by fission or emitting light particles passes the Coulomb barrier and is captured.

The fusion probabilities $T\left(J, E_{c m}\right)$ is assumed by 15$]$ as

$$
T\left(J, E_{c m}\right)=\sum_{V_{B}^{\prime}} f\left(V_{B}^{\prime}\right) t_{J}\left(E_{c m}, V_{B}^{\prime}\right)
$$

where $f\left(V_{B}^{\prime}\right)$ is a quasi-gaussian fusion barrier distribution, $t_{J}$ is the transmission coefficient obtained by WKB approximation. The fusion potential[14] is given by

$$
V_{R}=V_{\text {coul }}-V_{0} \exp \left[1.33\left(c_{p}+c_{t}-R\right) / b\right] c
$$

where $V_{\text {Coul }}$ is the Coulomb potential, $c_{p}$ and $c_{t}$ are the central radii [16] of projectile and target, $c=$ $c_{p} c_{t} /\left(c_{p}+c_{t}\right), \mathrm{b}$ is the surface diffuseness parameter [16]. The $V_{B}^{\prime}$ in eq.(2) is average barrier which was adjusted by a fixed constant $V_{0}$ (in $\mathrm{MeV} / \mathrm{fm}$ ) [17] in eq.(3). The fluctuation around $V_{B}^{\prime}$ is scaled by a parameter $r_{0}$. We have adopted a Gaussian distribution cut off at both ends at $r_{0} \pm t \sigma_{B}\left(r_{0}\right), \mathrm{t}$ is a cut-off parameter [15]. We use $\mathrm{t}$ $=3$ in our calculation. $\sigma_{B}\left(r_{0}\right)$ is standard deviation of $r_{0}$ distribution.

The level density is an important factor, the progress of synthesizing superheavy nucleus can be explained by the competition of fusion with a fast fission-like process which can be identified with quasi-fission 18]. Then the survival probability of residue evaporation nucleus can be expressed using the level densities in the compound and equilibrium configurations as [19]:

$$
P\left(J, E^{*}\right)=\frac{\rho\left(J, E_{c n}^{*}\right)}{\rho\left(J, E_{c n}^{*}\right)+\rho\left(J, E_{e q}^{*}\right)}
$$

where $\rho\left(J, E_{c n}^{*}\right)$ is the level density of compound configuration and $\rho\left(J, E_{e q}^{*}\right)$ is the level density of equilibrium 
configuration. The level density is

$$
\begin{aligned}
\rho\left(J, E^{*}\right) & =\frac{1}{24}\left(\frac{\hbar}{2 \theta}\right)^{3 / 2}(2 J+1) a^{1 / 2} U_{J}^{-2} \exp \left[2\left(a U_{J}\right)^{1 / 2}\right], \\
U_{J} & =E^{*}-E_{r}(J)+P_{\text {pair }}
\end{aligned}
$$

$P_{\text {pair }}$ is the pairing correction obtained from experimental odd-even mass fluctuations. $a$ is level density parameter, obtained from

$$
a=\bar{a}\left[1+f\left(E^{*}\right) B_{f}^{\text {shell }} / E^{*}\right]
$$

and

$$
f\left(E^{*}\right)=1-\exp \left(-E^{*} / E_{d}\right),
$$

in which $E_{d}$ is the damping energy, and

$$
\bar{a}=0.04543 r_{0}^{3} A+0.1355 r_{0}^{2} A^{2 / 3}+0.1426 r_{0} A^{1 / 3},
$$

where $E_{\gamma}(J)$ is the yrast energy of either the equilibrium configuration (light-particle and $\gamma$-emission) or the saddle-point configuration (fission), it reads

$$
E_{\gamma}(J)=J(J+1) \hbar^{2} / 2 \theta,
$$

in which $\theta$ is the moment of inertia. The fission barrier is defined by including the liquid drop component $\left(B_{f}^{L D}\right)$ and the shell component $\left(B_{f}^{\text {shell }}\right)$, scaled by a coefficient $C$, i.e,

$$
B_{f}=C\left(B_{f}^{L D}+B_{f}^{s h e l l}\right),
$$

in equilibrium configuration.

HIVAP takes into account the competition of $\gamma$-ray, neutron, proton and $\alpha$-particle emission with fission using angular-momentum and shape-dependent level densities and angular-momentum-dependent fission barriers.

The level densities have been calculated using the well known Fermi gas model. The same level density parameters have been used for the fission and neutron emission channels $\left(a_{f} / a_{n}=1\right)$. The arguments in support of this value were discussed in Ref. 20]. A phenomenological way of the introduction of shell effects into the level density calculation according to Ignatyuk 21], have been used in the evaporation channels. The shell damping energy $E_{d}$ is $18.5 \mathrm{MeV}$. The liquid-drop fission barrier $\left(B_{f}^{L D}\right)$ has been calculated according to the rotating charged liquid drop model of Cohen-Plasil-Swiatecki [22]. The shell component $\left(B_{f}^{\text {shell }}\right)$ of the fission barrier is equal to the difference between the liquid-drop model 23] and experimental [24] masses of the nucleus. Light-particle transmission coefficients are obtained using WKB approximation. The shell corrections are added at the saddle point.

In Fig.1 we use HIVAP model to calculate the fusion cross sections for the reaction of ${ }^{18} \mathrm{O}+{ }^{248} \mathrm{Cm}$. The cross sections for the channels with $3 \mathrm{n}$ to $7 \mathrm{n}$ emission

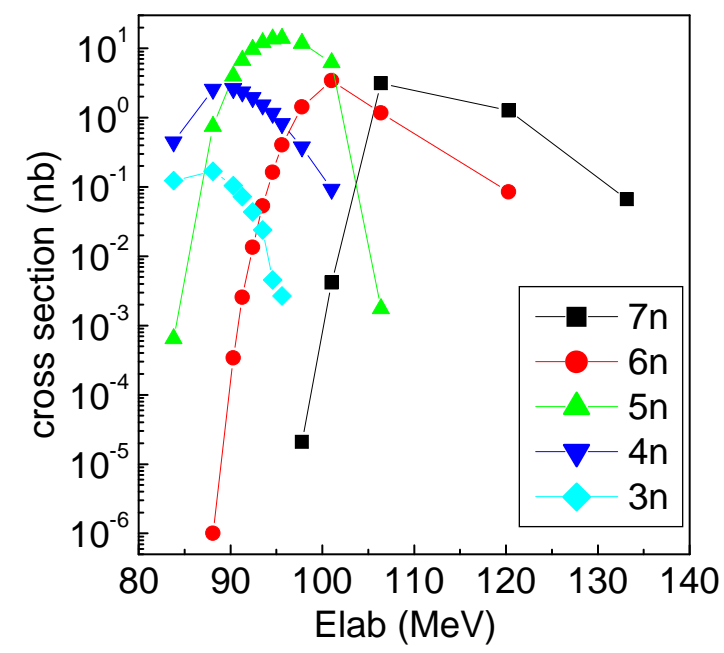

FIG. 1: Calculated evaporation residue cross sections for ${ }^{18} \mathrm{O}+{ }^{248}$ $\mathrm{Cm}$ in different xn channels.

are shown. Generally the energy corresponding to the peak of the cross section for a given xn channel increases with the number of emitted neutrons, which is consistent with the excitation extent of the reaction with the more neutron emission. In all channels, the maximum cross section can be reached for $5 \mathrm{n}$ channels. Since ${ }^{18} \mathrm{O}$ $+{ }^{248} \mathrm{Cm}$ is a hot fusion reaction, we take the maximum value of cross section at $5 \mathrm{n}$ channel to compare with the experimental data [26, 27, 30]. Fig. 2 shows the comparison. An overall satisfied overlap of the calculations with the data was obtained. We listed the experiment data in Table I. From the comparison, it looks that this model has a good agreement with the data for these reactions.

So far, we used the HIVAP to fit the experimental data very well. Based on this achievement, we would like to make some predictions for the synthesizing the elements of $\mathrm{Z}=109$. With all the same parameters in the model calculation, we calculated some channels for producing $\mathrm{Z}=109$ elements and listed the results is in Table II. From this table, we found that the channels of ${ }^{30} \mathrm{Si}+{ }^{243}$ $\mathrm{Am} \rightarrow{ }^{270} \mathrm{Mt}+3 n$ at $\mathrm{E}=151 \mathrm{MeV}$ or ${ }^{30} \mathrm{Si}+{ }^{243} \mathrm{Am} \rightarrow{ }^{269}$ $M t+4 n$ at $\mathrm{E}=161 \mathrm{MeV}$ have a larger cross sections for synthesizing the new isotopes of element 109 . The cross section can reach to $\simeq 21 \mathrm{pb}$.

From the calculation results listed in Table 1 we can see the maximum error of our calculations is within 5 times comparing with the experimental data, so it seems that our estimation for cross section should be reasonable. Element 109 was produced firstly in cold fusion by physicists of the Heavy Ion Research Laboratory, Darmstadt, West Germany using ${ }^{58} \mathrm{Fe}$ ion bombarding on ${ }^{209} \mathrm{Bi}$ 




FIG. 2: Comparison the calculation results by HIVAP (line) with the experimental data (symbols). The different symbols present the results from the different works which have been already listed in Table I.

TABLE I: The calculation of cross sections for synthesizing $\mathrm{Z}=103-107$ calculate by HIVAP. "*" represent the reaction which has been finished in LanZhou (HIRFL) recently but no data published yet. The result which calculated by Alice code is $1.8 \mathrm{nb}$ [25].

\begin{tabular}{ccccc}
\hline \hline channel & $\begin{array}{c}E_{\text {Lab }} \\
(\mathrm{MeV})\end{array}$ & $\begin{array}{c}\sigma_{\text {exp }} \\
(\mathrm{nb})\end{array}$ & Reference & $\begin{array}{c}\text { HIVAP } \\
(\mathrm{nb})\end{array}$ \\
\hline${ }^{248} \mathrm{Cm}\left({ }^{18} \mathrm{O}, 5 \mathrm{n}\right){ }^{261} \mathrm{Rf}$ & 94.2 & $<0.9$ & {$[27]$} & \\
${ }^{248} \mathrm{Cm}\left({ }^{18} \mathrm{O}, 5 \mathrm{n}\right){ }^{261} \mathrm{Rf}$ & 97 & 5 & {$[26]$} & 13 \\
${ }^{248} \mathrm{Cm}\left({ }^{18} \mathrm{O}, 5 \mathrm{n}\right){ }^{261} \mathrm{Rf}$ & 100.4 & $4.5_{-2.0}^{+3.5}$ & {$[27]$} & 12 \\
${ }^{248} \mathrm{Cm}\left({ }^{18} \mathrm{O}, 5 \mathrm{n}\right){ }^{261} \mathrm{Rf}$ & 103.9 & $1.7-1.3$ & {$[27]$} & 4.4 \\
\hline${ }^{248} \mathrm{Cm}\left({ }^{18} \mathrm{O}, 5 \mathrm{n}\right){ }^{261} \mathrm{Rf}$ & 91 & $8 \pm 2$ & {$[30]$} & 4.2 \\
${ }^{248} \mathrm{Cm}\left({ }^{18} \mathrm{O}, 5 \mathrm{n}\right){ }^{261} \mathrm{Rf}$ & 94 & $13 \pm 3$ & {$[30]$} & 11.3 \\
${ }^{248} \mathrm{Cm}\left({ }^{18} \mathrm{O}, 5 \mathrm{n}\right){ }^{261} \mathrm{Rf}$ & 99 & $8 \pm 2$ & {$[30]$} & 15 \\
\hline${ }^{244} \mathrm{Pu}\left({ }^{22} \mathrm{Ne}, 5 \mathrm{n}\right){ }^{261} \mathrm{Rf}$ & 114 & 4.4 & {$[28]$} & 7.8 \\
${ }^{244} \mathrm{Pu}\left({ }^{22} \mathrm{Ne}, 5 \mathrm{n}\right){ }^{261} \mathrm{Rf}$ & 120 & 3.8 & {$[28]$} & 4.6 \\
\hline${ }^{248} \mathrm{Cm}\left({ }^{19} \mathrm{~F}, 5 \mathrm{n}\right){ }^{262} \mathrm{Db}$ & 106 & 2 & {$[29]$} & \\
${ }^{248} \mathrm{Cm}\left({ }^{19} \mathrm{~F}, 5 \mathrm{n}\right){ }^{262} \mathrm{Db}$ & 106 & $1.3 \pm 0.4$ & {$[30]$} & 0.67 \\
${ }^{248} \mathrm{Cm}\left({ }^{19} \mathrm{~F}, 5 \mathrm{n}\right){ }^{262} \mathrm{Db}$ & 106.5 & $0.26{ }_{-0.09}^{+0.15}$ & {$[31]$} & \\
\hline${ }^{248} \mathrm{Cm}\left({ }^{15} \mathrm{~N}, 5 \mathrm{n}\right){ }^{258} \mathrm{Lr}$ & 88 & 200 & {$[32]$} & 480 \\
\hline${ }^{248} \mathrm{Cm}\left({ }^{15} \mathrm{~N}, 4 \mathrm{n}\right){ }^{259} \mathrm{Lr}$ & 80 & 40 & {$[32]$} & 169 \\
\hline${ }^{249} \mathrm{Cf}\left({ }^{18} \mathrm{O}, 4 \mathrm{n}\right){ }^{263} \mathrm{Sg}$ & 95 & 1 & {$[33]$} & 2.3 \\
\hline${ }^{249} \mathrm{Cf}\left({ }^{15} \mathrm{~N}, 4 \mathrm{n}\right){ }^{260} \mathrm{Db}$ & 84 & 3.5 & {$[34]$} & 13 \\
\hline${ }^{243} \mathrm{Am}\left({ }^{18} \mathrm{O}, 5 \mathrm{n}\right){ }^{261} \mathrm{Lr}$ & 96 & 30 & {$[35]$} & 149 \\
\hline${ }^{249} \mathrm{Bk}\left({ }^{15} \mathrm{~N}, 4 \mathrm{n}\right){ }^{260} \mathrm{Rf}$ & 82 & 18 & {$[36]$} & 31 \\
\hline${ }^{249} \mathrm{Bk}\left({ }^{18} \mathrm{O}, 5 \mathrm{n}\right){ }^{262} \mathrm{Db}$ & 99 & $6 \pm 3$ & {$[37]$} & 2.1 \\
\hline${ }^{241} \mathrm{Am}\left({ }^{22} \mathrm{Ne}, 4 \mathrm{n}\right){ }^{259} \mathrm{Db}$ & 118 & $1.6 \pm 1.2$ & {$[38]$} & 2.7 \\
\hline${ }^{243} \mathrm{Am}\left({ }^{26} \mathrm{Mg}, 4 \mathrm{n}\right){ }^{265} \mathrm{Db}$ & 131 & $\mathrm{~N} / \mathrm{A}$ & $*$ & 0.097 \\
\hline \hline
\end{tabular}

TABLE II: The calculated cross section for synthesizing element 109 .

\begin{tabular}{|c|c|c|}
\hline Channel & $E_{l a b}(\mathrm{MeV})$ & Cross section $(\mathrm{pb})$ \\
\hline${ }^{254} \mathrm{Es}\left({ }^{22} \mathrm{Ne}, 4 \mathrm{n}\right){ }^{272} \mathrm{Mt}$ & 115 & 8 \\
\hline${ }^{254} \mathrm{Es}\left({ }^{22} \mathrm{Ne}, 5 \mathrm{n}\right){ }^{271} \mathrm{Mt}$ & 124 & 5 \\
\hline${ }^{254} \mathrm{Es}\left({ }^{20} \mathrm{Ne}, 4 \mathrm{n}\right){ }^{270} \mathrm{Mt}$ & 114 & 3 \\
\hline${ }^{254} \mathrm{Es}\left({ }^{20} \mathrm{Ne}, 5 \mathrm{n}\right){ }^{269} \mathrm{Mt}$ & 120 & 3 \\
\hline${ }^{249} \mathrm{Bk}\left({ }^{26} \mathrm{Mg}, 4 \mathrm{n}\right){ }^{271} \mathrm{Mt}$ & 137 & 9.5 \\
\hline${ }^{249} \mathrm{Bk}\left({ }^{26} \mathrm{Mg}, 5 \mathrm{n}\right){ }^{270} \mathrm{Mt}$ & 148 & 5 \\
\hline${ }^{243} \mathrm{Am}\left({ }^{28} \mathrm{Si}, 4 \mathrm{n}\right){ }^{267} \mathrm{Mt}$ & 155 & 3 \\
\hline${ }^{243} \mathrm{Am}\left({ }^{30} \mathrm{Si}, 4 \mathrm{n}\right){ }^{269} \mathrm{Mt}$ & 161.3 & 13 \\
\hline${ }^{243} \mathrm{Am}\left({ }^{30} \mathrm{Si}, 3 \mathrm{n}\right){ }^{270} \mathrm{Mt}$ & 151 & 21.6 \\
\hline
\end{tabular}

target. For element 109, the isotopes of ${ }^{268} \mathrm{Mt}$ and ${ }^{266} \mathrm{Mt}$ were already synthesized in GSI [39]. We suggest that the channel of ${ }^{30} \mathrm{Si}+{ }^{243} \mathrm{Am} \rightarrow{ }^{270} \mathrm{Mt}+3 \mathrm{n}$ or ${ }^{30} \mathrm{Si}+{ }^{243} \mathrm{Am} \rightarrow{ }^{269} \mathrm{Mt}+4 n$ to synthesize new isotopes of element $109 .{ }^{30} \mathrm{Si}$ is a suitable projectile since there is $3.1 \%{ }^{30} \mathrm{Si}$ contained in element $\mathrm{Si}$ and it is a stable nucleus. Considering the present situations of our national laboratory in Lanzhou (HIRFL), it is very difficult to synthesize the very heavy elements such as above $\mathrm{Z}=$ 112 etc. in the moment, but it is feasible to try to search for the element 109 with the proposed reaction channel.

To recap, the extensive experimental data for the synthesis of superheavy nuclei have been well reproduced in our calculations with help of the HIVAP code. It illustrates that the statistical-evaporation model can still work for the production of superheavy nuclei in a suitable mass region. Within a reasonable extrapolation, we propose to use a new channel, i.e. ${ }^{30} \mathrm{Si}+{ }^{243} \mathrm{Am} \rightarrow{ }^{270}$ $M t+3 n$ or ${ }^{30} S i+{ }^{243} A m \rightarrow{ }^{269} M t+4 n$ to synthesize new isotopes of element 109.

Ma Y G would like to appreciate Dr. Martin Veselsky for providing HIVAP code and worthy discussions.

* Supported by the Major State Basic Research Development Program under Contract No G200077400, the National Natural Science Foundation of China under (NNSFC) Grant No 10135030, and the CAS Grant for Distinguished Young Scholars of NNSFC under Grant No 19725521.

$\dagger$ To whom correspondence should be addressed. Email: ygma@sinr.ac.cn

[1] Oganessian Y T, Yeremin A V et al. 1999 Nature 400 242; Oganessian Y T, Utyonkov V K et al. 1999 Phys. Rev. Lett. 833154.

[2] Oganessian Y T, Utyonkov V K et al. 2001 Phys. Rev. C 63011301.

[3] Ren Z Z, Tai F, Chen DH 2002 Phys. Rev. C 66064306.

[4] Bao J D et al. 2002 Nucl. Phys. A 70747.

[5] Wang N, Zhao E G, Li J F et al. 2003 Chin Phys Lett 
20635.

[6] Wang N, Wu X Z, Li Z X 2003 Chin. Phys. Lett. 201466.

[7] Zhang W, Zhang S S, Zhang S Q, Meng J 2003 Chin. Phys. Lett. 201694.

[8] Wu Z Y, Xu F R, Zhao E G, Zheng C K 2003 Chin. Phys. Lett. 201702.

[9] Chen B Q, Ma Z Y, Zhao Y L 2003 Chin. Phys. Lett. 20 1936.

[10] Reisdorf W 1981 Z. Phys. A. 300227.

[11] Reisdorf W 1992 Z. Phys. A. 34347.

[12] Andreyev A N et al 1997 Nucl. Phys. A. 626857.

[13] Veselsky M et al 1997 Z. Phys. A. 356403.

[14] Reisdorf W et al 1985 Nucl. Phys. A. 444154.

[15] Reisdorf W et al 1985 Nucl. Phys. A438 212.

[16] Blocki J et al. 1977 Ann. of Phys. 105427.

[17] Myers W D Schmidt K H. 1983 Ncll. Phys. A410 61.

[18] Shen W Q et al. 1987 Phys Rev C 36115.

[19] Veselsky M 1999 Acta. Phys. Slovaca. 49101.

[20] Andreyev A N et al. 1997 Nucl. Phys. A 620229.

[21] Ignatyuk A V, Smirenkin G N Tishin A S 1975 Yad. Fiz. 21485.

[22] Cohen S, Plasil F, Swiatecki W J 1974 Ann. Phys. 82 557.

[23] Myers W D, Swiatecki W J 1974 Ann. Phys. 82557.

[24] Wapstra A H et al. 1988 Data. Nucl. Data. Tabl. 39281.
[25] Gan Z G, Guo J S et al. 2003 Nucl. Phys. Rev. 20 99;

[26] Ghiorso A et al 1970 Phys. Lett. 32B 95.

[27] Dressler R 1999 PhD Thesis University of Berm.

[28] Lazarev Yu A et al 2000 Phys. Rev. C 6206307.

[29] Naour C Le, Trubert D et al. 1999 Extended Abstracts of the 1st International Conference on the Chemistry and Physics of the Transactinide Elements, Seeheim, Germany.O-12Sep. 26.

[30] Nagame Y et al 2002 J. Nucl. Rad. Sci. 3.185.

[31] Dressler R Eichler B Jost K T et al 1999 Phys. Rev. C 593433.

[32] Eskola K, Eskola P et al 1971 Phys. Rev. C. 4632.

[33] Ghiorso A, Nitschke J M et al 1974 Phys. Rev. Lett. 33 1490.

[34] Druin V A, Bochev B et al 1979 Sov. J. Nucl. Phys.29 591.

[35] Flerov G N et al 1967 Nucl. Phys. A106 476.

[36] Gnitschke J M, Fowler M et al 1981 Nucl. Phys. A. 352 138.

[37] Ghiorso A et al 1970 Phys. Lett. 32B 95.

[38] Guo J S, Qin Z, Gan Z G et al 2002 Jour. of. Nucl. and. Radi. Scie. 3183.

[39] Münzenberg, G Reisdorf, W Hofmann and S Agarwal Y K 1984 Z. Phys. A 315145. 